\title{
COVID-19-Associated Coagulopathy in the Peripartum Setting: A Case Report
}

\author{
Ashley Allen, DO ${ }^{1} \quad$ Christine Hoang, $\mathrm{MD}^{1} \quad$ Roopina Sangha, MD ${ }^{1}$ \\ ${ }^{1}$ Department of Obstetrics and Gynecology, John Peter Smith \\ Hospital, Fort Worth, Texas

\begin{abstract}
Address for correspondence Ashley Allen, DO, Department of Obstetrics \& Gynecology, John Peter Smith Hospital, 1500 S Main St, Fort Worth, TX 76104 (e-mail: aatheobg@gmail.com).
\end{abstract}

AJP Rep 2022;12:e33-e35.

\begin{abstract}
Keywords

- COVID-19

- coagulopathy

- D-dimer

- peripartum

- anticoagulation

Sepsis-induced coagulopathy (SIC) scoring and D-dimer can be used to recognize COVID-19induced coagulopathy, but the utility of these is largely unknown in the peripartum setting and leaves anticoagulation guidance unclear. We present the case of a critically ill postpartum patient with COVID-19 infection. This patient presented with clinical signs of COVID-19 infection and developed acute respiratory failure requiring invasive mechanical ventilation and subsequent cesarean delivery at 34 weeks. She initially improved postoperatively but deteriorated after postoperative day 5 . She was found to have a very elevated D-dimer of $58 \mu \mathrm{g} / \mathrm{mL}$ and anticoagulation was escalated to full dosing. She required prolonged mechanical ventilation and deceased after developing gram-positive cocci bacteremia. This case demonstrates that recognition and management of COVID-19associated coagulopathy can be confusing in the peripartum period and studies are needed to validate D-dimer and SIC scoring in this population of patients.
\end{abstract}

The sepsis-induced coagulopathy (SIC) scoring system is a validated tool developed by the International Society of Thrombosis and Haemostasis (ISTH) to facilitate early recognition of disseminated intravascular coagulopathy (DIC). The SIC scoring has also been used to identify coagulopathy in patients with COVID-19. In a retrospective analysis of 449 COVID-19 patients that met SIC criteria or had a markedly elevated D-dimer (>3.0 $\mu \mathrm{g} / \mathrm{mL}$ ), a $20 \%$ decrease in 28-day mortality was demonstrated in patients receiving prophylactic low-molecularweight heparin (LMWH) or unfractionated heparin. ${ }^{2}$ The utility of D-dimer and SIC scoring tool has not been validated in the peripartum setting but may still be valuable in recognizing COVID-19-associated coagulopathy as demonstrated in our case.

\section{Case}

A 30-year-old inmate and Native American G6P5 at 34 weeks of gestation presented to the emergency room on March 31,
2020 with a cough worsening over 2 weeks. Associated symptoms included fatigue and nausea. She was afebrile but did have tachypnea at 22 respirations per minute and oxygen saturation at $94 \%$ on room air. Chest X-ray showed a questionable infiltrate in the periphery of the right lower lobe. Initial laboratory work was significant for absolute lymphopenia at $7,700 \times 10^{3} / \mu \mathrm{L}, \mathrm{C}$-reactive protein (CRP) 8.0 $\mathrm{mg} / \mathrm{dL}$, ferritin $72 \mathrm{ng} / \mathrm{mL}$, and procalcitonin $0.11 \mathrm{ng} / \mathrm{mL}$. Initial blood cultures were negative. A nasopharyngeal swab for SARS-CoV-2 by polymerase chain reaction (PCR) was obtained. She was started on azithromycin and ceftriaxone. Her pregnancy was complicated by a history of five prior cesarean sections. She had been recently transferred to Fort Worth, Texas, from a federal facility in South Dakota 2 weeks preceding her admission.

On April 1, 2020, while being monitored on the labor and delivery unit, her tachypnea worsened and she developed profound dyspnea with ambulation. She required $4 \mathrm{~L} / \mathrm{min}$ by nasal cannula to maintain oxygen saturation above $95 \%$. received

September 23, 2020 accepted after revision October 8, 2021
DOI https://doi.org/

10.1055/s-0041-1742237. ISSN 2157-6998.
(C) 2022. The Author(s).

This is an open access article published by Thieme under the terms of the Creative Commons Attribution-NonDerivative-NonCommercial-License, permitting copying and reproduction so long as the original work is given appropriate credit. Contents may not be used for commercial purposes, or adapted, remixed, transformed or built upon. (https://creativecommons.org/ licenses/by-nc-nd/4.0/)

Thieme Medical Publishers, Inc., 333 Seventh Avenue, 18th Floor, New York, NY 10001, USA 
She also developed a fever to $38.3^{\circ} \mathrm{C}$. A course of hydroxychloroquine was initiated in conjunction with continued azithromycin. Her respiratory status continued to worsen, so the decision was made to intubate and mechanically ventilate. There was subsequent concern for fetal distress and the decision was made to proceed with delivery. The SARS-CoV-2 PCR result was still pending at this time, but given the high suspicion for the disease, antenatal steroids for neonatal benefit were deferred for concern of potential maternal harm. She had an uncomplicated c-section with a quantitative blood loss of $450 \mathrm{~mL}$. Tranexamic acid was given intraoperatively. The infant was intubated for respiratory distress and sent to the neonatal intensive care unit (NICU). Cord blood gas analysis showed a mild respiratory acidosis, arterial $\mathrm{pH} 7.13, \mathrm{pO}_{2} 24$, and $\mathrm{pCO}_{2} 79$ with a base deficit of 4.7 $\mathrm{mmol} / \mathrm{L}$. The infant tested negative for SARS-CoV-2 PCR. The infant was extubated the next day and was stable on room air by the 4 th day of life. The infant discharged home with a family member on the 18th day of life.

On postoperative day 3 , a positive SARS-CoV-2 PCR resulted. Application for remdesivir was approved on postoperative day 5 and initiated as $200 \mathrm{mg}$ once followed by $100 \mathrm{mg}$ daily for 10 days, and she completed the antiviral course.

Ventilator settings were consistent with ARDSnet protocol guidelines. Ventilation was initially done using assist control (AC)/volume control (VC) with a gradually rising $\mathrm{PaO}_{2} / \mathrm{FiO}_{2}$ ratio up to 440 by postoperative day 5 . Her $\mathrm{PaO}_{2} / \mathrm{FiO}_{2}$ ratio dropped to 232 on postoperative day 6 and she was transitioned to airway pressure release ventilation (APRV). Of note, her CRP was 12 but increased to 33 on postoperative day 8 , and she reached a maximum temperature of $39.9^{\circ} \mathrm{C}$. She developed a superficial wound separation 1 week postoperatively and a vacuum-assisted closure device was placed, which was changed every 3 to 4 days depending on positioning. Several attempts were made to wean paralytics; however, it consistently resulted in prolonged episodes of hypoxia. She remained in the prone position and was intermittently supinated as tolerated.

A bronchoscopy was done on postoperative day 12 due to refractory hypoxia, and findings were concerning for diffuse alveolar hemorrhage (DAH). DAH workup including deoxyribonucleic acid (DNA) double-strained (DS) antibodies, antineutrophil cytoplasmic antibody (ANCA) vasculitis panel, and glomerular basement membrane antibodies were negative. Tocilizumab was given the same day. The following day, a blood clot developed in her radial artery catheter. A D-dimer resulted as $58 \mu \mathrm{g} / \mathrm{mL}$. She had a platelet count of $413 \times 10^{9} / \mathrm{L}$, international normalized ratio (INR) of 1.29 , and a Sequential Organ Failure Assessment (SOFA) score of 4 equating to an SIC score of 3. The decision was made to transition to full anticoagulation using a heparin drip with a goal titration partial thromboplastin time (PTT) of 50 to 80 seconds. Cardiolipin antibodies were negative.

Upon availability, she received convalescent plasma on postoperative day 20. She developed gram-positive cocci bacteremia and deceased after 28 days of mechanical ventilation.

\section{Conclusion}

An important mechanism of COVID-19-associated mortality is diffuse alveolar damage, small vessel thrombosis, and thrombotic microangiopathy as demonstrated in autopsies of COVID-19 patients. ${ }^{3}$ Connors and Levy perspective on anticoagulation management in COVID-19 findings conclude that coagulopathy is related to severity of illness and resultant thromboinflammation as opposed to viral activity. ${ }^{4}$ It is well known that procoagulation factors are increased in pregnancy, resulting in a fivefold increased risk of thromboembolism when compared with nonpregnant women and 60-fold increased risk in the first 3 months after delivery. ${ }^{5}$ These findings suggest that pregnant and postpartum patients may be more susceptible to the thromboinflammatory effects of COVID-19 resulting in worsening illness. This is demonstrated in our case given the deterioration after postoperative day 5 . Similar findings are reflected in a case series of two antepartum patients admitted for severe COVID-19 who underwent c-section. Both patients deteriorated on postoperative day 3 and had a D-dimer greater than $2 \mu \mathrm{g} / \mathrm{mL}$. They improved after receiving full dose anticoagulation.

Interestingly, a person's risk of severe illness from COVID-19 is very similar to the individual risk factors for venous thromboembolism (VTE) in pregnancy. This list includes heart disease, obesity, autoimmune disease, hypertension, and sickle cell disease. Cesarean delivery is also an independent risk factor for VTE with an estimated fourfold increased risk as compared with vaginal delivery. ${ }^{6}$ Cesarean delivery has not been listed as a risk factor for severe illness in COVID-19 infection but, as demonstrated in our case as well as in the aforementioned two case series, it may potentially worsen maternal risk of serious illness particularly when the inflammatory response is at its peak around postoperative days 3 to 5 . This suggestion is validated in a cohort of 82 women with a positive SARS-CoV-2 reverse transcription PCR (RT-PCR) and who delivered within 14 days of admission; $13.5 \%$ of women undergoing c-section had severe maternal outcomes and 21\% had clinical deterioration. Some women who underwent cesarean delivery may have had the confounding factor of preterm delivery for worsening maternal disease, but even after adjusting for this confounder, cesarean delivery remained independently associated with increased risk of clinical deterioration. ${ }^{7}$ This may suggest that women admitted for moderate to severe COVID-19 infection and presence of other risk factors for VTE, including cesarean delivery, should at minimum receive prophylactic anticoagulation and may benefit from escalation to intermediate or full dosing especially if the D-dimer or SIC score is elevated.

We suggest considering use of the SIC score or a markedly elevated D-dimer for early recognition of COVID-19-associated coagulopathy in the peripartum setting. Patients with an elevated SIC score or markedly elevated D-dimer should receive therapeutic anticoagulation as in our case. Despite not having validation of D-dimer or SIC scoring in pregnant or postpartum women, this should not preclude them from aggressive anticoagulation as their risk of VTE and development of severe COVID-19 illness is likely higher than nonpregnant patients. 


\section{Conflict of Interest}

Dr Roopina Sangha declares the following conflict of interest: received payment or honoraria for lectures, presentations, speakers bureaus, manuscript writing, or educational events from John Peter Smith Hospital, Fort Worth, Texas.

\section{References}

1 Iba T, Levy JH, Warkentin TE, Thachil J, van der Poll T, Levi MScientific and Standardization Committee on DIC, and the Scientific and Standardization Committee on Perioperative and Critical Care of the International Society on Thrombosis and Haemostasis. Diagnosis and management of sepsis-induced coagulopathy and disseminated intravascular coagulation. J Thromb Haemost 2019;17(11):1989-1994

2 Tang N, Bai H, Chen X, Gong J, Li D, Sun Z. Anticoagulant treatment is associated with decreased mortality in severe coronavirus disease 2019 patients with coagulopathy. J Thromb Haemost 2020;18(05):1094-1099

3 Fox SE, Akmatbekov A, Harbert JL, Li G, Quincy Brown J, Vander Heide RS. Pulmonary and cardiac pathology in African American patients with COVID-19: an autopsy series from New Orleans. Lancet Respir Med 2020;8(07):681-686

4 Connors JM, Levy JH. COVID-19 and its implications for thrombosis and anticoagulation. Blood 2020;135(23):2033-2040

5 Pomp ER, Lenselink AM, Rosendaal FR, Doggen CJ. Pregnancy, the postpartumperiod andprothromboticdefects: riskofvenous thrombosis in the MEGA study. J Thromb Haemost 2008;6(04): 632-637

6 Blondon M, Casini A, Hoppe KK, Boehlen F, Righini M, Smith NL. Risks of venous thromboembolism after cesarean sections: a meta-analysis. Chest 2016;150(03):572-596

7 Martínez-Perez O, Vouga M, Cruz Melguizo S, et al. Association between mode of delivery among pregnant women with COVID19 and maternal and neonatal outcomes in Spain. JAMA 2020;324 (03):296-299 